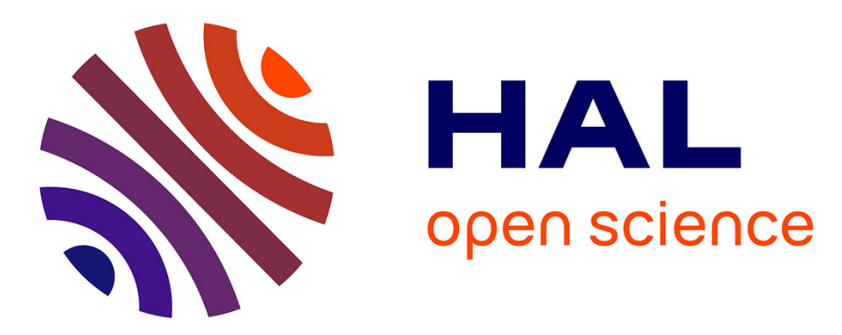

\title{
Characterization of soliton compounds in a passively mode-locked high power fiber laser
}

Foued Amrani, Mohamed Salhi, Hervé Leblond, François Sanchez

\section{To cite this version:}

Foued Amrani, Mohamed Salhi, Hervé Leblond, François Sanchez. Characterization of soliton compounds in a passively mode-locked high power fiber laser. Optics Communications, 2010, 283 (24), pp.5224 - 5230. 10.1016/j.optcom.2010.08.003 . hal-03187581

\section{HAL Id: hal-03187581 \\ https://univ-angers.hal.science/hal-03187581}

Submitted on 1 Apr 2021

HAL is a multi-disciplinary open access archive for the deposit and dissemination of scientific research documents, whether they are published or not. The documents may come from teaching and research institutions in France or abroad, or from public or private research centers.
L'archive ouverte pluridisciplinaire HAL, est destinée au dépôt et à la diffusion de documents scientifiques de niveau recherche, publiés ou non, émanant des établissements d'enseignement et de recherche français ou étrangers, des laboratoires publics ou privés. 
Provided for non-commercial research and education use. Not for reproduction, distribution or commercial use.

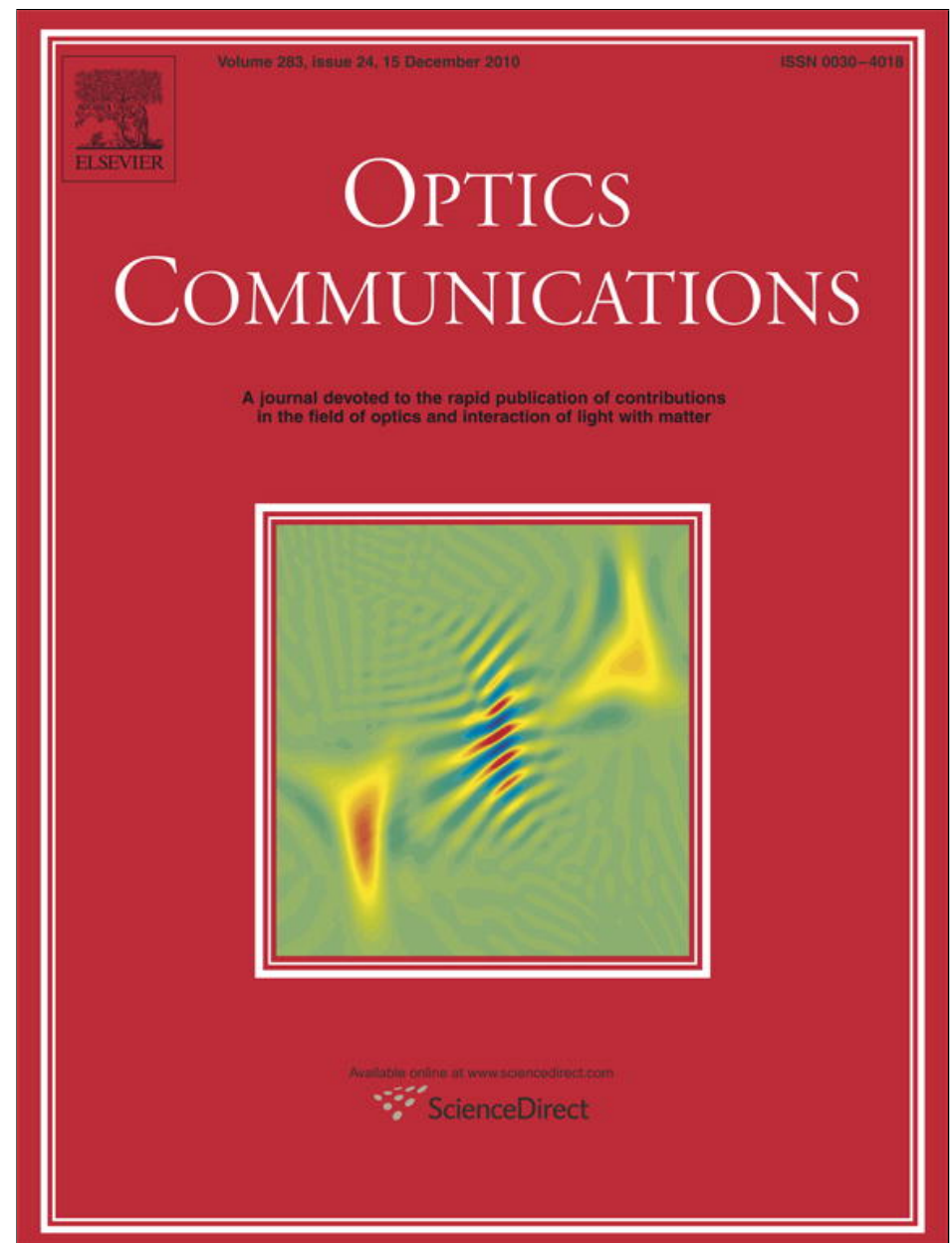

This article appeared in a journal published by Elsevier. The attached copy is furnished to the author for internal non-commercial research and education use, including for instruction at the authors institution and sharing with colleagues.

Other uses, including reproduction and distribution, or selling or licensing copies, or posting to personal, institutional or third party websites are prohibited.

In most cases authors are permitted to post their version of the article (e.g. in Word or Tex form) to their personal website or institutional repository. Authors requiring further information regarding Elsevier's archiving and manuscript policies are encouraged to visit:

http://www.elsevier.com/copyright 


\title{
Characterization of soliton compounds in a passively mode-locked high power fiber laser
}

\author{
Foued Amrani, Mohamed Salhi, Hervé Leblond, François Sanchez* \\ Laboratoire de Photonique d'Angers EA 4644, Université d'Angers 2 Bd Lavoisier, 49000 Angers, France
}

\section{A R T I C L E I N F O}

\section{Article history:}

Received 18 June 2010

Received in revised form 20 July 2010

Accepted 3 August 2010

\begin{abstract}
A B S T R A C T
We characterize soliton complexes in a high power double-clad erbium-doped fiber laser passively modelocked through nonlinear polarization evolution. Such complexes involve some hundreds of solitons and form self-organized or disorganized patterns analogous to the states of the matter. Experimentally these soliton compounds are characterized through the autocorrelation trace, the optical spectrum and the oscilloscope trace which is limited due to its finite bandwidth. We perform here a reconstruction of the experimental results thus allowing us to identify the temporal distribution of the solitons inside the cavity. The reconstruction allows us to clarify and either to confirm or to correct the former intuitive interpretation. Especially, a soliton 'spray' is identified.
\end{abstract}

(C) 2010 Elsevier B.V. All rights reserved.

\section{Introduction}

Multiple pulsing is a very usual behaviour in passively modelocked fiber lasers operating in the anomalous dispersion regime [15]. Indeed, the quantization of the soliton energy favours the emergence of several pulses by cavity round-trip. With standard pumping powers, the number of solitons is limited. As a consequence of the soliton interaction, pulses lead to different pattern formation along the cavity. The pattern can be disordered [6] or completely organized [7] including passive harmonic mode-locking [8,9]. When the solitons fill all the available space along the cavity in a disordered way, the distribution of the solitons along the cavity is easily observable with classical detection apparatus. Organized structures correspond to soliton compounds in which few identical pulses remain at the same distance from each other and with a constant dephasing from one round-trip to the other $[10,11]$. Such states can be completely characterized through the measurement of both the optical spectrum and autocorrelation trace. From these measurements, one can completely retrieve the temporal distribution of the solitons which cannot be directly observed because of the small temporal delays between neighbouring pulses $[11,12]$. This problem of retrieving the soliton distribution becomes more complex with high power fiber lasers, especially with condensate phases where hundreds of solitons are grouped in a small part of the cavity [13-17]. Inside a condensate phase, solitons can move or can be at rest. In the

\footnotetext{
* Corresponding author. Tel.: +33 241735 447; fax: + 33241735216 E-mail address: francois.sanchez@univ-angers.fr (F. Sanchez).
}

latter case, the soliton distribution can be more or less regular. Experimentally we have access to the envelop of the soliton train, to the optical spectrum and only to a limited part of the autocorrelation trace. With a limited number of pulses this is sufficient to deduce the temporal distribution of the solitons without any further calculations. When hundreds of pulses are present, the reconstruction is not straightforward and requires a specific study. In a recent paper [17], we reported a close analogy with the states of the matter and soliton compounds in an erbium-doped double-clad fiber laser passively mode-locked by nonlinear polarization rotation. Indeed, the laser delivers some hundreds of solitons by cavity round-trip resulting in spontaneous pattern formation as a consequence of the soliton interaction. In view of the optical spectra and autocorrelation traces, we proposed a temporal distribution for the solitons. However, we did not check in detail that the suggested distributions were fully compatible with the experimental results. The aim of this paper is to perform a reconstruction of the experimental data reported in Ref. [17].

The starting point is a given temporal distribution for the electric field including many solitons. We consider successively the case of a soliton 'gas', a soliton 'liquid', a soliton 'poly-crystal' and a soliton 'crystal'. All the states are experimentally obtained by adjusting adequately the orientation angle of an intra-cavity phase plate. In each case of interest we propose an ansatz and calculate the corresponding optical spectrum and autocorrelation trace. In order that the conditions are the same as in the experiment, the results are averaged over several cavity round trips. The averaging is limited to 4 roundtrip because the results do not vary beyond this value. The reconstructed results are in good agreement with the experimental acquisitions thus allowing evidencing the temporal distribution of the solitons along the cavity. 


\section{Soliton 'gas'}

In this section we consider a state which occurs very frequently and consists of an assembly of solitons filling all the available space along the cavity and in perpetual relative movement, without any phase correlation between the pulses and from one round-trip to the other. For the reconstruction we consider the following ansatz:

$E(t)=\sum_{k=1}^{N} A_{0} \operatorname{sech}\left(\frac{t_{k}}{\tau}\right) \exp i\left(-C \frac{t_{k}^{2}}{2 \tau^{2}}+\omega_{0} t_{k}+\phi_{k}\right)$,

where $t_{k}=t-\sum_{\ell=1}^{k} \Delta \tau_{\ell} . \Delta \tau_{k}$ is the temporal delay between pulse $k$ and pulse $k-1$ except for $k=1$ for which it corresponds to the temporal position of the first soliton, chosen as the origin of times $t=0 . \phi_{k}$ is the phase of the $k$ th soliton. For the 'gas' state $\Delta \tau_{k}$ and $\phi_{k}$ are considered as random variables from one round-trip to the other and also from one pulse to the other. $\phi_{k}$ is uniformly distributed in $[0, \pi]$, and $\Delta \tau_{k}$ over all the sites of numerical box. $\tau$ is related to the pulse duration and is assumed to be the same for all the pulses. Its value is deduced experimentally from the autocorrelation trace as $\tau=0.7 \mathrm{ps}$. $C$ is the chirp parameter and is fixed in order to adjust the width of the reconstructed optical spectrum to its experimental value. Examples of temporal distributions are given in Fig. 1 in which each curve corresponds to a different round-trip. In order to reduce the calculation time, we have considered only 50 pulses and a reduced temporal window of $3.2 \mathrm{~ns}$ while the experimental values are 500 and $105 \mathrm{~ns}$, respectively. This has neither consequence on the reconstructed optical spectrum nor on the autocorrelation trace. Indeed, we have checked that no change occurs by increasing the number of pulses and/or the width of the temporal window. Hence our choice is a good compromise between a reasonable calculation time and negligible consequences on the reconstructed results.

The optical spectrum is numerically calculated as the Fourier transform of the electric field (1) and then averaged over several cavity round trips in order to mimic the operating conditions of an optical spectrum analyzer. The autocorrelation trace is determined by

$I(T)=\int_{-\infty}^{+\infty}|E(t) E(t+T)|^{2} d t$

which corresponds to the non collinear configuration as it was used in our experiment. Here again, the trace is averaged over several round trips to reproduce the experimental conditions.
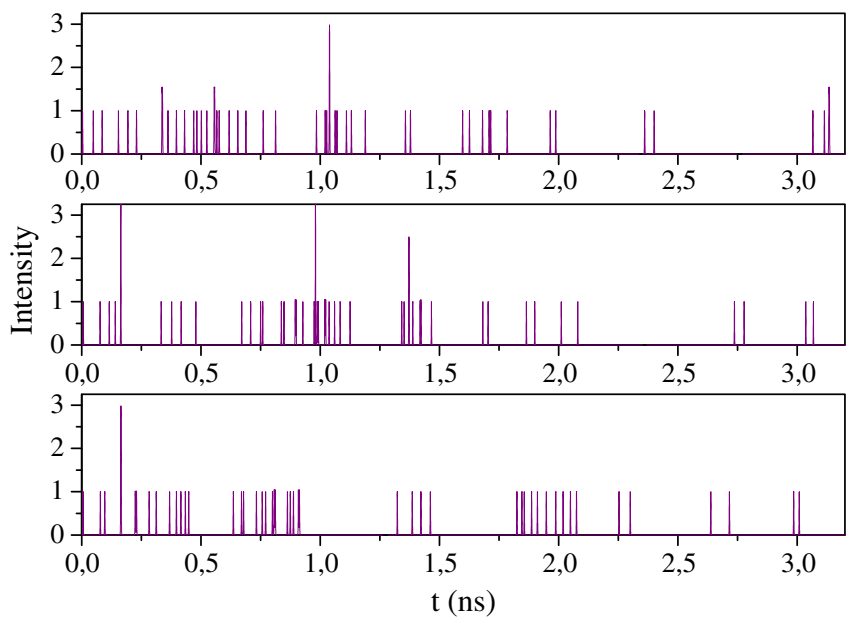

Fig. 1. Reconstructed time distribution of the intensity associated to a soliton 'gas'. The different curves correspond to different round-trip.
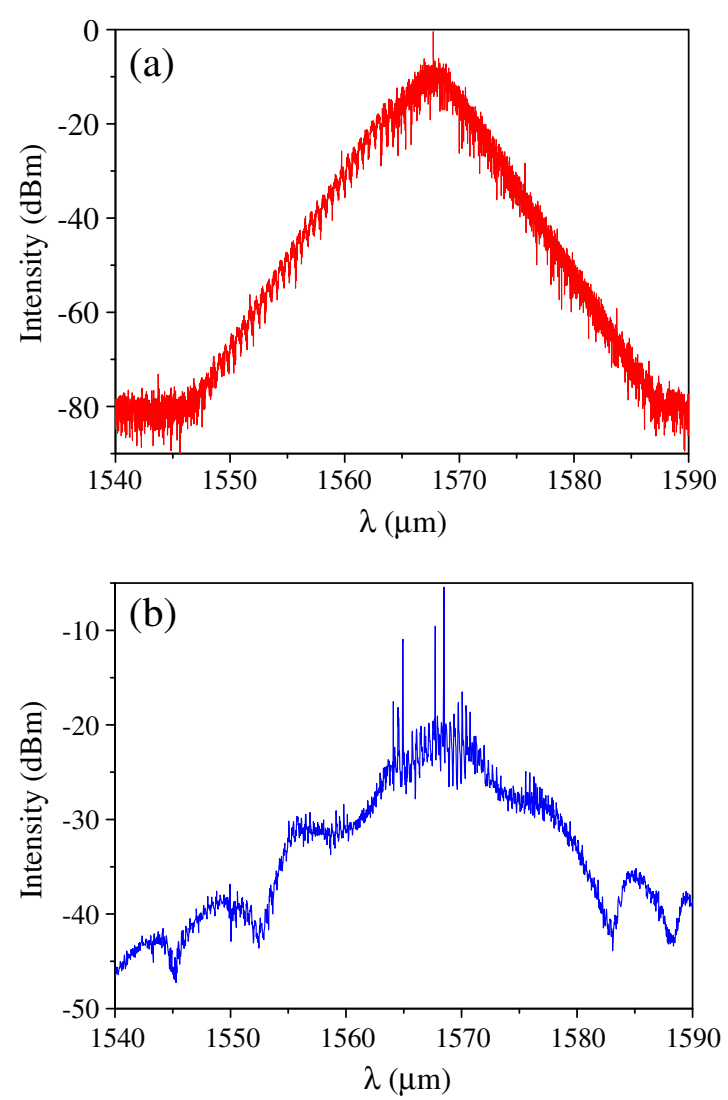

Fig. 2. Optical spectrum for the soliton 'gas'. (a) Reconstructed and (b) experimental.
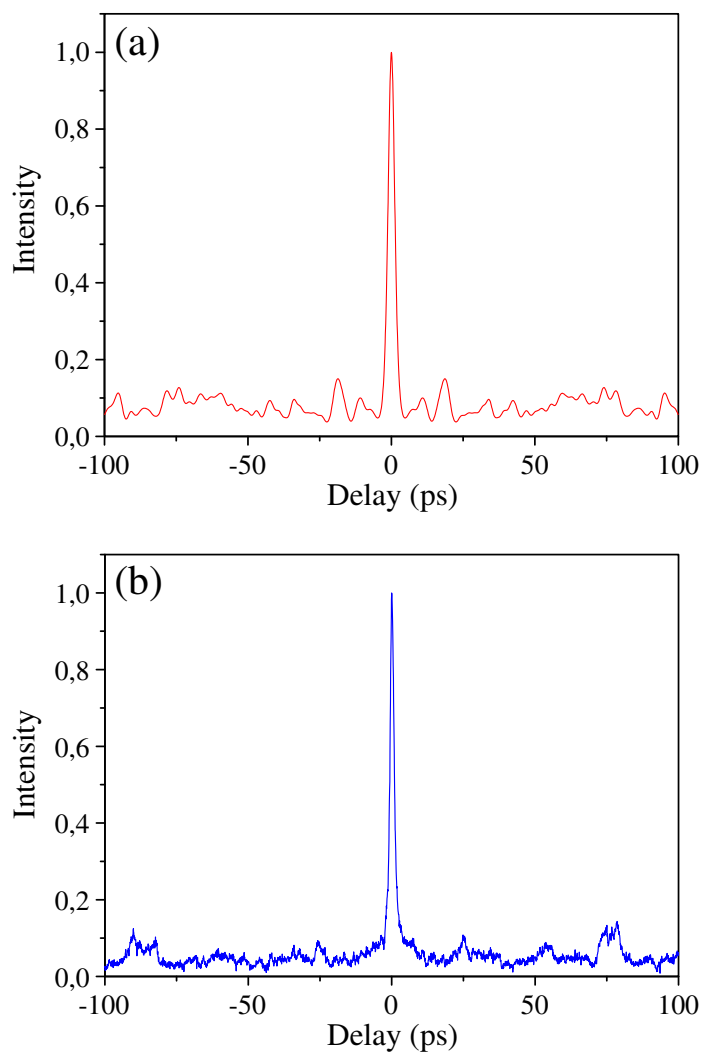

Fig. 3. Autocorrelation trace for the soliton 'gas'. (a) Reconstructed and (b) experimental. 
The comparison between the reconstructed results and the experimental acquisitions is given in Figs. 2 and 3. The chirp parameter is here $\mathrm{C}=0.7$. Both the reconstructed optical spectrum and autocorrelation trace compare favourably with the experimental results, at least in what concerns the general shape of the spectrum and its fast modulation. The fact that there is no mutual coherence between the pulses results in both the absence of modulation in the spectrum and the absence of intercorrelation peaks in the autocorrelation trace. Moreover, the large pedestal in the autocorrelation trace is related to the perpetual movement of the solitons. Hence the reconstruction confirms that the experimental results correspond to a temporal distribution given by relation (1).

\section{Soliton 'liquid'}

The usual definition of a liquid assumes that the distance between particles is close to some fixed value $\Delta \tau_{0}$, with a small jitter. In the case
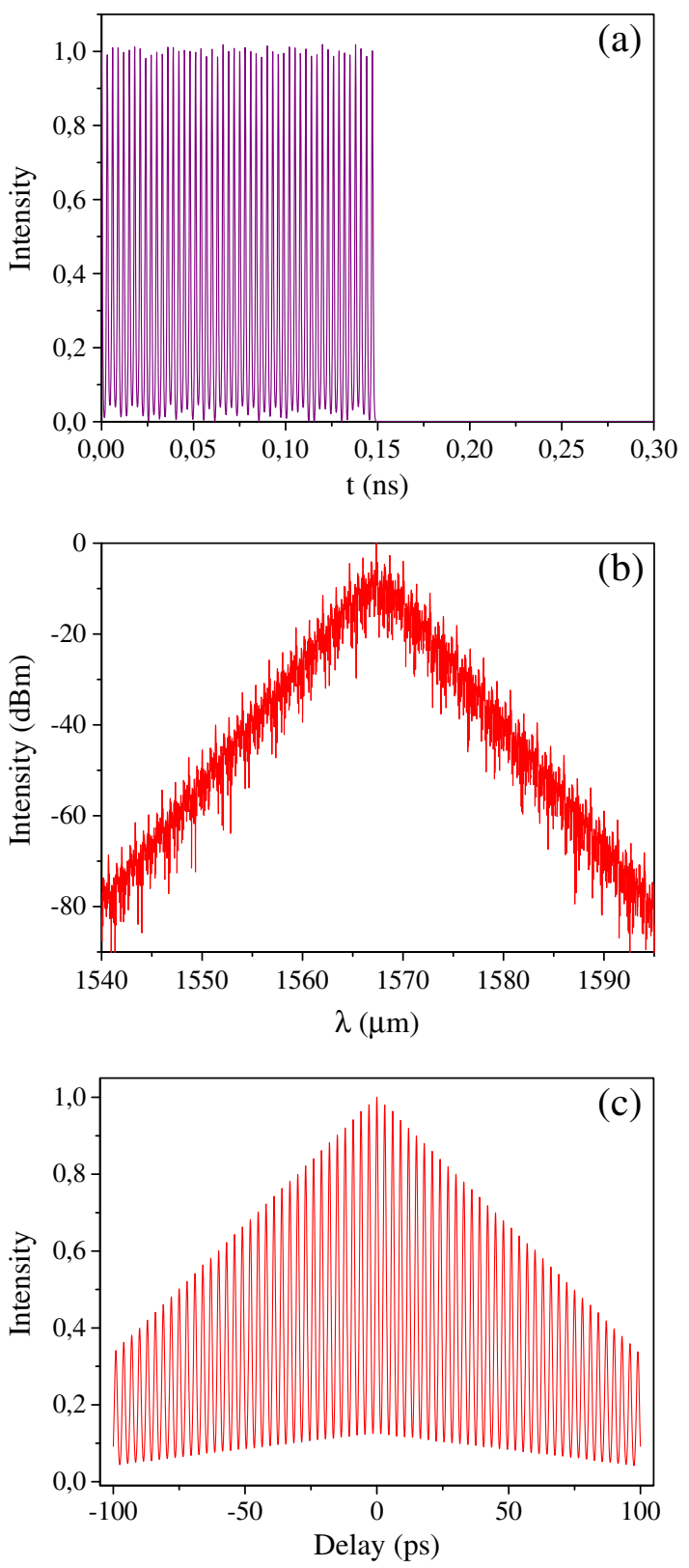

Fig. 4. The reconstructed soliton 'liquid', as defined by ansatz (3). a) Temporal distribution, b) optical spectrum, c) autocorrelation trace.

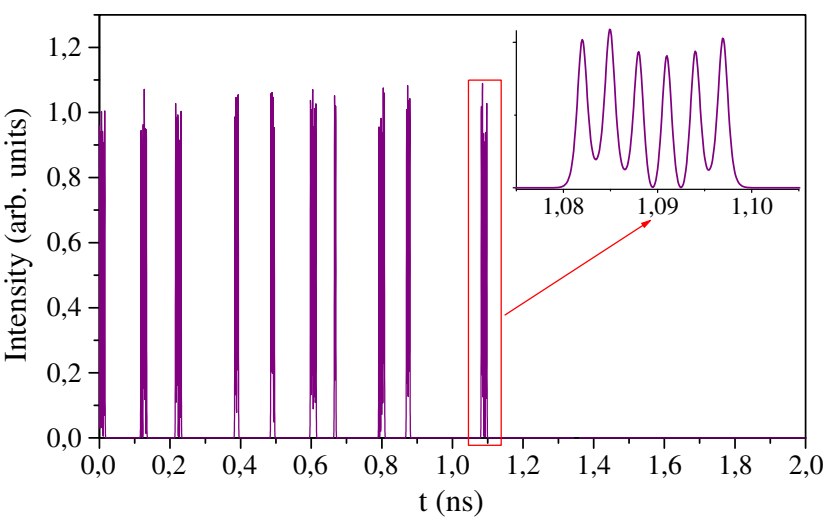

Fig. 5. Temporal distribution of a soliton 'spray'. The inset shows a zoom of the selected cluster.

of solitons, the non-rigid character of the inter-particle bounds in a 'liquid' corresponds to the fact that no specific phase relation between neighbouring solitons is present. This can be described by the ansatz

$E(t)=\sum_{k=1}^{N} A_{0} \operatorname{sech}\left(\frac{t_{k}}{\tau}\right) \exp i\left(-C \frac{t_{k}^{2}}{2 \tau^{2}}+\omega_{0} t_{k}+\phi_{k}\right)$

with $t_{k}=t-\sum_{\ell=1}^{k}\left(\Delta \tau_{0}+\varepsilon_{\ell}\right)$, where $\varepsilon_{\ell} \ll \Delta \tau_{0}$ varies randomly from one pulse to the other. More precisely, $\varepsilon_{\ell}=0.05 \cos w$, with $w$ distributed uniformly in $[0, \pi]$. The phase $\phi_{k}$ is also random, distributed uniformly in $[0, \pi]$. The reconstruction based on ansatz (3) yields the temporal distribution, optical spectrum and autocorrelation trace shown on Fig. 4. For the numerical computations we have used the parameters $\tau=0.7 \mathrm{ps}, \mathrm{C}=0.8$ and $\Delta \tau_{0}=3 \mathrm{ps}$ (they correspond to the experimental values)
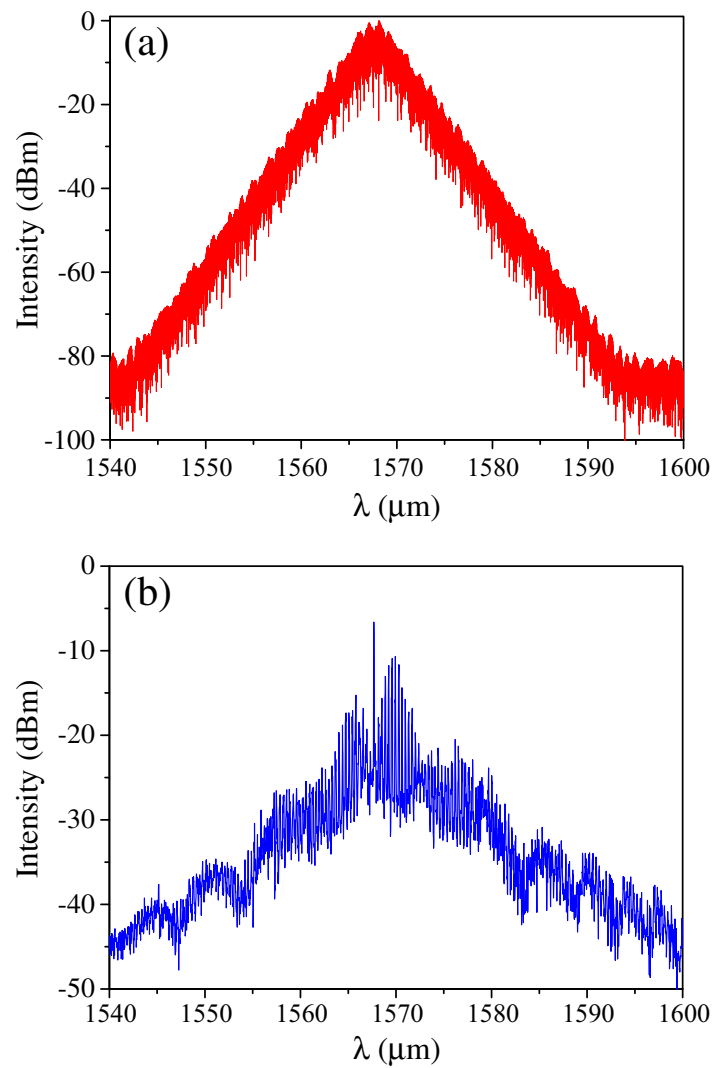

Fig. 6. Optical spectrum for the soliton 'spray'. (a) Reconstructed and (b) experimental. 


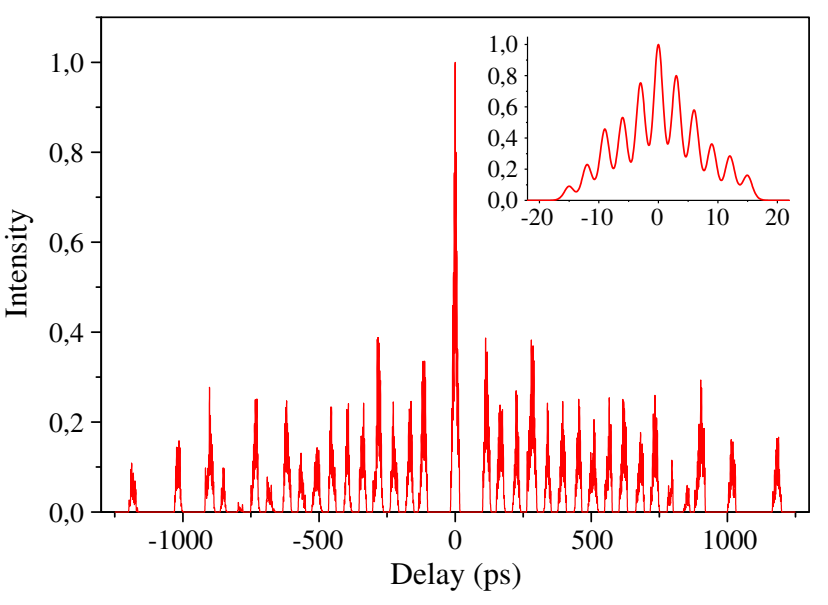

Fig. 7. Reconstructed autocorrelation trace of the soliton 'spray'. The inset shows a zoom of the central part.

An experimentally observed multisoliton state was called soliton 'liquid' in Ref. [17]. The experimental data did not show long-range order, while the reconstruction does, which proves that ansatz (3) does not represent the observed situation. The fact that the usual definition of a liquid induces here long-range correlation is due to dimensionality. More precisely, fixing with some precision the distance between neighbouring particles does not fix at all the distance between two particles at long range in 2 or 3 dimensions. In contrast to this, in the present case, $t_{k}=t-k \Delta \tau_{0}+\eta_{k}$, in which the random variable $\eta_{k}=\sum_{\ell=1}^{k} \varepsilon_{\ell}$, has a variance of about $\varepsilon \sqrt{k}$, in which we denote by $\varepsilon$ is the common variance of the $\varepsilon_{\ell}$. This induces long-range order as long as $\varepsilon \sqrt{k}$ remains smaller than $\Delta \tau_{0}$. As a conclusion, what we called soliton 'liquid' in Ref. [17] is not a liquid in the usual sense corresponding to ansatz (3).

Reconsidering the experimental observations, which show a small modulation in the optical spectrum together with several peaks in the autocorrelation trace, suggests that there are some clusters of solitons in relative movement rather than only isolated moving solitons. For the reconstruction we consider a further ansatz, which consists in an incoherent superposition of clusters containing
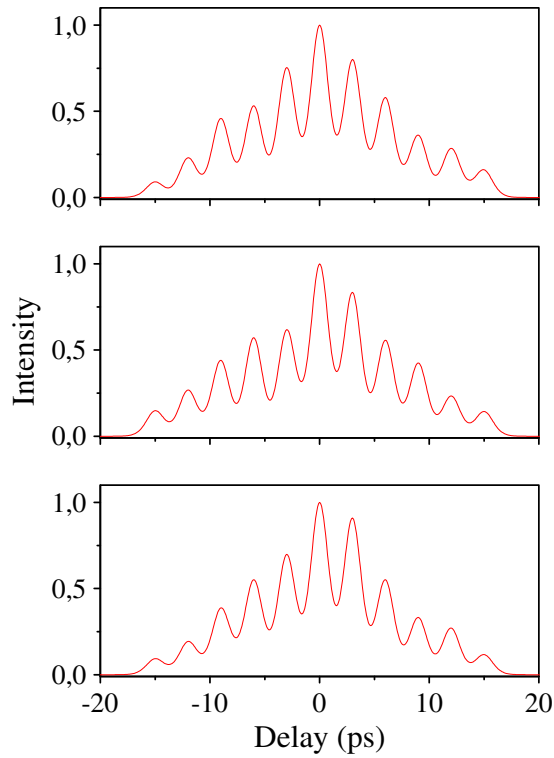

a variable number of pulses. The total electric field is built as the sum of $\mathrm{N}$ different clusters

$E(t)=\sum_{n=1}^{N} E_{n}\left(t-\sum_{j=1}^{n} \Delta T_{j}\right)$,

where $\Delta T_{j}$ is the delay between two successive clusters. The electric field for the cluster $\mathrm{n}$ is written as

$E_{n}(t)=\sum_{\ell=1}^{\ell_{n}} A_{0} \operatorname{sech}\left(\frac{t_{\ell, n}}{\tau}\right) \exp i\left(-C \frac{t_{\ell, n}^{2}}{2 \tau^{2}}+\omega_{0} t_{\ell, n}+\phi_{\ell, n}\right)$,

where

$t_{\ell, n}=t-\sum_{m=1}^{\ell} \Delta \tau_{m, n}$

$\Delta \tau_{m, n}=\Delta \tau_{0}+\varepsilon_{m, n}$

The parameters $\Delta T_{j}, \varepsilon_{m, n}$ and $\phi_{\ell, n}$ are considered as random variables, with $\left|\varepsilon_{m, n}\right| \ll \Delta \tau_{0} \ll \Delta T_{j}$.

No phase correlation between solitons is assumed, hence $\phi_{\ell, n}$ varies randomly inside a cluster (as $\ell$ varies), from a cluster to the other (as $n$ varies) and from one round-trip to the other. The separation time between two clusters $\Delta T_{j}$ is random, while the position of a soliton in a cluster is close to a fixed equilibrium value $\Delta \tau_{0}$, with a small jitter, varying randomly as much as $\phi_{\ell, n}$ does. The $\phi_{\ell, n}$ are distributed uniformly in $[0, \pi], \Delta T_{j}$ uniformly over the sites of the computation box, and $\varepsilon_{m, n}=0.05 \cos w$ with $w$ distributed uniformly in $[0, \pi]$ as above. For the numerical computations we used $\tau=0.7 \mathrm{ps}$ (measured experimental value) and a chirp parameter $\mathrm{C}=0.8$. The latter is slightly different from the one used for the soliton 'gas'. This is compatible with theoretical results reported in Ref. [18] which show that the chirp depends on the orientation of the phase plates. An example of temporal distribution is given in Fig. 5.

The optical spectrum is given in Fig. 6. The reconstructed spectrum is in good agreement with the experimental one: a weak modulation occurs because of the clustering effect between solitons. An example of reconstructed autocorrelation trace is shown in Fig. 7. Because of the large temporal distribution of the solitons in comparison with the
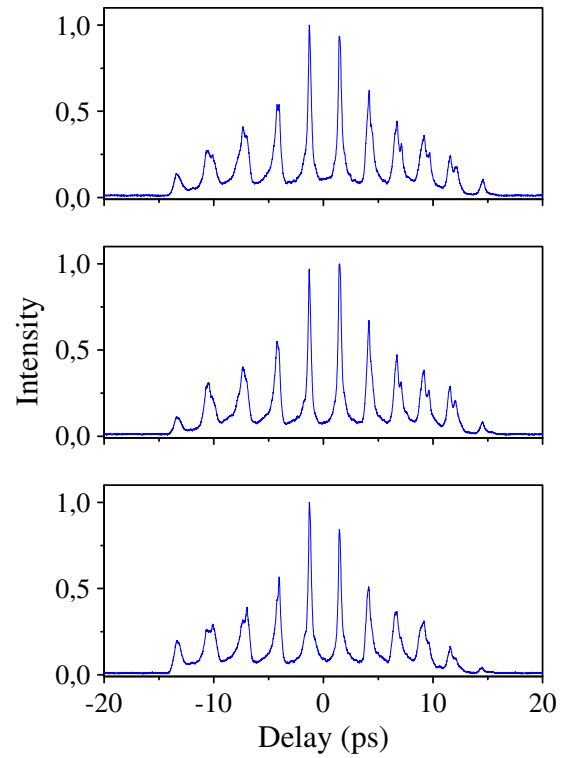

Fig. 8. Autocorrelation trace of the soliton ‘spray'. Left: reconstructed, right: experimental. The three reconstructed traces correspond to three different drawings of random variables. The three experimental traces correspond to three successive acquisitions. 


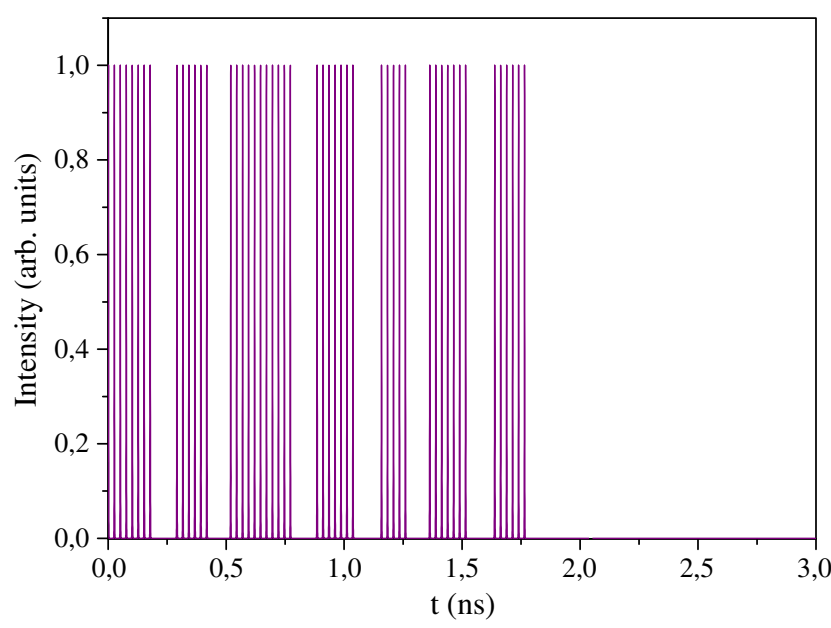

Fig. 9. Temporal distribution of a soliton 'poly-crystal'.

pulse duration, the autocorrelation trace exhibits many peaks. Experimentally we have access only to a small region around the zero delay. As a consequence, the comparison with experimental data will be done in this reduced region. Results are compared in Fig. 8 for several initial acquisition times. The reconstructed traces perfectly reproduce the pedestal and the various peaks. In addition, the autocorrelation trace changes depending on the exact initial time of acquisition as it is the case experimentally. The good agreement between the reconstructed results and the experimental data
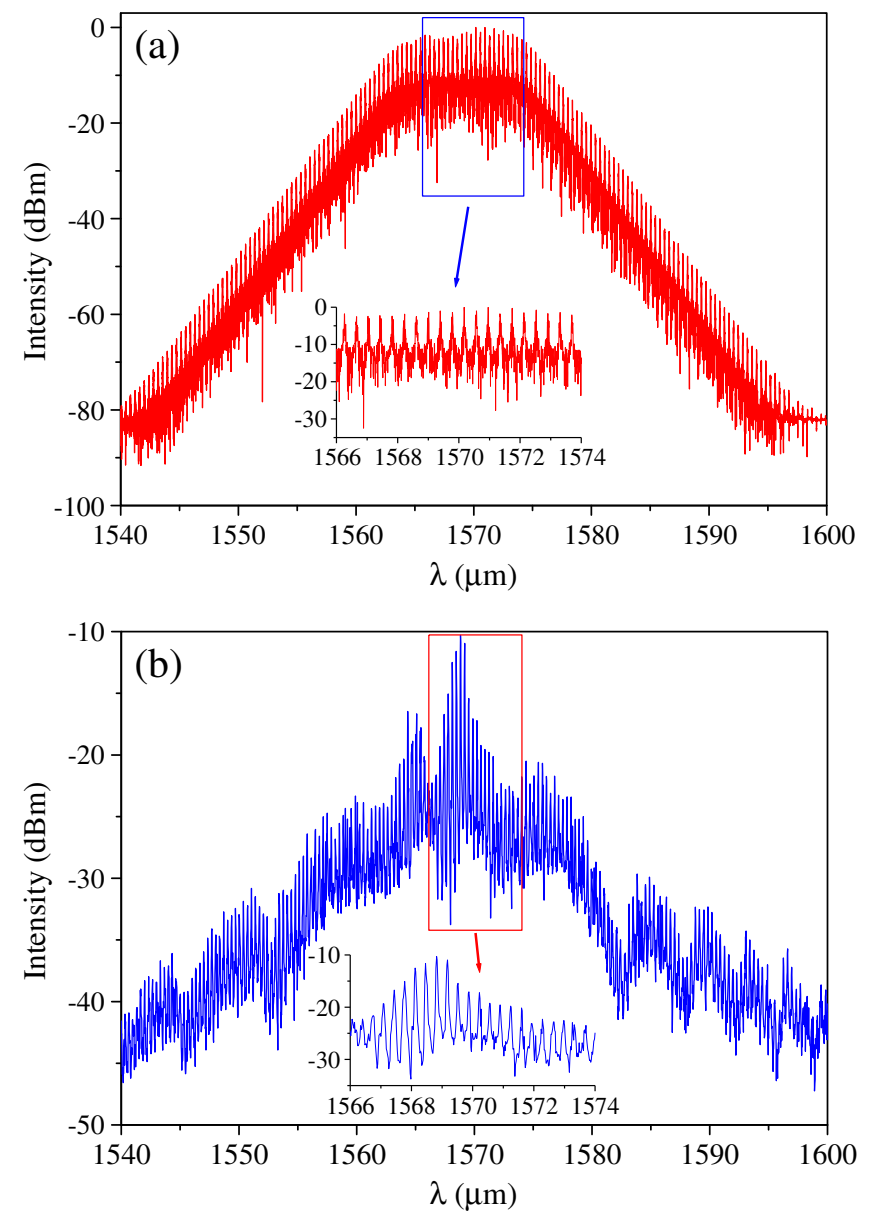

Fig. 10. Optical spectrum for the soliton 'poly-crystal'. (a) Reconstructed and (b) experimental.
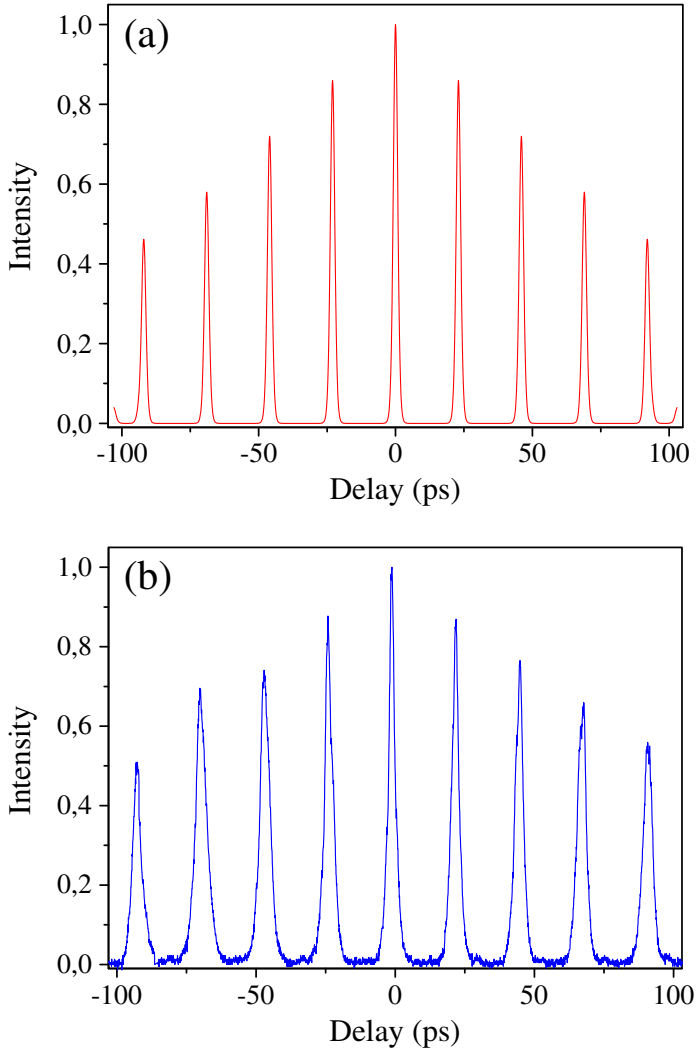

Fig. 11. Autocorrelation trace for the soliton 'poly-crystal'. (a) Reconstructed and (b) experimental.

confirms that the experimental state corresponds to an incoherent superposition of soliton clusters. As a conclusion, the state we called previously a soliton 'liquid' should rather be called a 'spray' or a 'fog' of solitons because it is analogous to a suspension of micro-droplets.

\section{Soliton 'poly-crystal'}

This experimental state is a condensate phase in which the solitons fill only a part of the cavity and the distribution repeats from one round-trip to the other. The optical spectrum has a significant modulation and the autocorrelation trace exhibits some sharp peaks

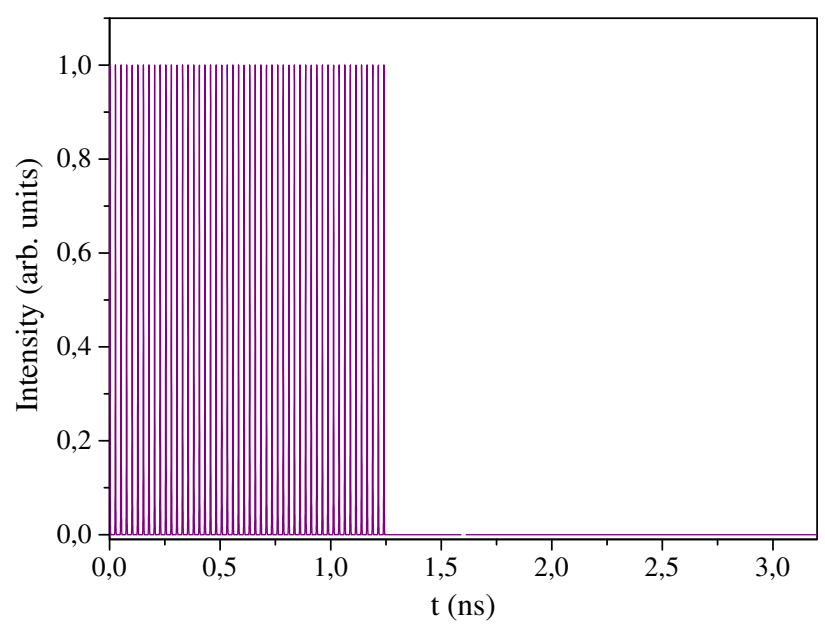

Fig. 12. Temporal distribution of a soliton 'crystal'. 

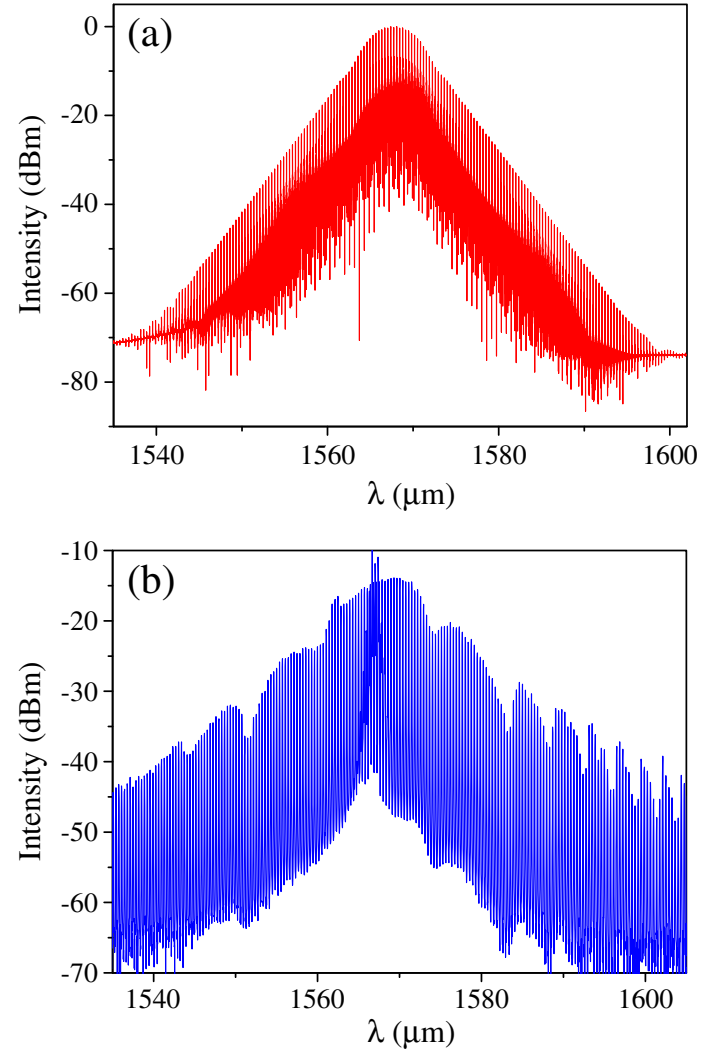

Fig. 13. Optical spectrum for the soliton 'crystal'. (a) Reconstructed and (b) experimental.

without any pedestal. These observations led us to interpret such state as a soliton 'poly-crystal', i.e. an incoherent superposition of clusters in the form of bound states. To confirm this point of view we consider an electric field distribution similar to Eqs. (4) and (5) except that $\Delta \tau_{m, n}=\Delta \tau_{0}$ for all $\mathrm{m}, \mathrm{n}$ and $\phi_{\ell, n}=\phi_{n}$. The first relation means that the time delay between neighbouring solitons is invariant all along the 'poly-crystal', while the second relation means that there is a constant phase relation between solitons inside a cluster but a random relative phase between different clusters. This random phase is distributed uniformly over $[0, \pi] . \Delta T_{j}$ is a random variable, distributed uniformly over the sites of the numerical box, but remains constant from one round-trip to the other. An example of temporal distribution of the solitons is given in Fig. 9. For the computations we have used $\tau=0.9 \mathrm{ps}$ and $\mathrm{C}=1$.

The optical spectrum is shown in Fig. 10. Results show that the reconstructed spectrum exhibits a significant modulation in reasonable agreement with the experimental observations (see insets, which show the regularity of the modulation). The spectral period depends only on the delay $\Delta \tau_{0}$. As in the case of a soliton 'liquid', the autocorrelation trace spreads over a large temporal range which cannot be observed experimentally. As a consequence we restrict the reconstructed traces to the range experimentally achievable, i.e. $\pm 100 \mathrm{ps}$. Results are given in Fig. 11. Here again the reconstruction favourably compares with the experiment: the trace has a nearly triangular envelop as it has with a bound state and no pedestal. The reconstruction therefore demonstrates that the experimental observations are compatible with a temporal distribution consisting in an incoherent mixture of bound states repeating from one round-trip to the other.

\section{Soliton 'crystal'}

The last state reported in Ref. [17] consisted in a bound state of some hundred solitons. This state was completely characterized in Refs. $[13,15]$. However, in order to give a complete overview we give here the reconstruction. The ansatz consists in a coherent superposition of $\mathrm{N}$ identical solitons. The solitons are equidistant and fill only a part of the cavity:

$E(t)=\sum_{\zeta=1}^{N} A_{0} \operatorname{sech}\left(\frac{t-(\ell-1) \Delta \tau}{\tau}\right) \operatorname{expi}\left(-C \frac{(t-(\ell-1) \Delta \tau)^{2}}{2 \tau^{2}}+\omega_{0}(t-(\ell-1) \Delta \tau)\right) \cdot(8)$

For the numerical computations, we have used the experimental pulse duration $\tau=0.9 \mathrm{ps}$ and delays $\Delta \tau=23 \mathrm{ps}$, the chirp parameter is $C=1.2$. An example of temporal distribution is shown in Fig. 12 .

The optical spectra are shown in Fig. 13. The reconstructed spectrum exhibits the same characteristics than the experimental one: there is a strong and regular modulation meaning that the solitons are completely coherent and equidistant. Fig. 14 shows the autocorrelation traces. The reconstructed one has a triangular envelop because solitons form a bound state. In the reduced range experimentally achievable, the variations of the amplitude of the peaks are not appreciable. Experimentally these variations are below the noise level and the autocorrelation trace exhibits nearly constant regularly spaced peaks. Figs. 13 and 14 show that there is a very good agreement between the reconstructed results and the experimental data.

\section{Conclusion}

In conclusion, we have fully characterized the different soliton complexes observed experimentally in the erbium-doped double-clad fiber laser. For that we have performed a reconstruction based on an
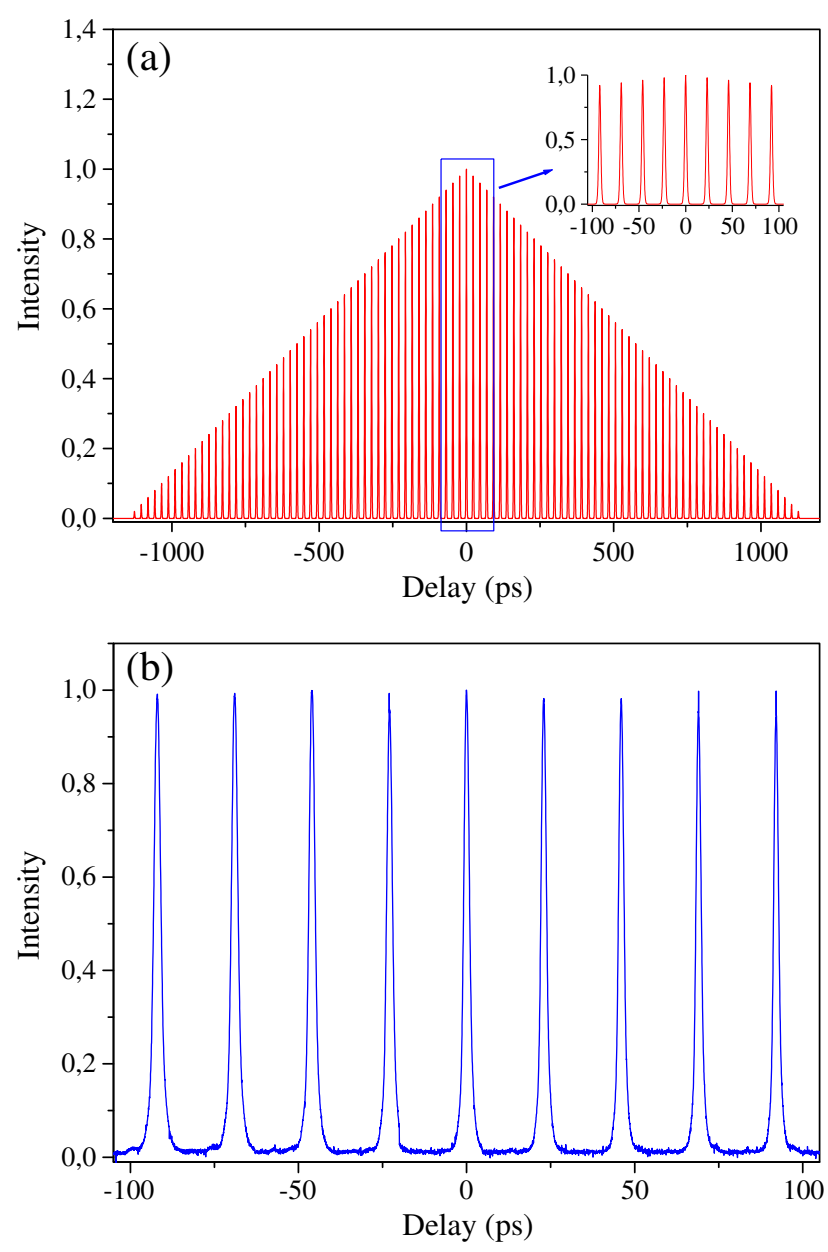

Fig. 14. Autocorrelation trace for the soliton 'crystal'. (a) Reconstructed and (b) experimental. 
adequate ansatz for the electric field distribution inside the laser cavity. The calculated optical spectra and autocorrelation traces are in good agreement with their corresponding experimental results, which demonstrates the validity of the reconstruction. The interpretation of the experimental results of Ref. [17] is confirmed for the soliton 'gas', 'crystal', and 'poly-crystal' states. The more accurate analysis shows that the state which was previously thought to be a 'liquid' is in fact a 'spray', while the characteristics of the 'liquid' are well determined. We expect to observe the soliton 'liquid' state in some future experiment, since it has not yet been observed.

\section{References}

1] Ph. Grelu, J.M. Soto-Crespo, J. Opt. B: Quant. Semiclass. Opt. 6 (2004) S271

2] D.Y. Tang, W.S. Man, H.Y. Tam, Opt. Commun. 165 (1999) 189.

3] A. Komarov, H. Leblond, F. Sanchez, Phys. Rev. A 71 (2005) 053809.

4] A. Haboucha, A. Komarov, H. Leblond, M. Salhi, F. Sanchez, J. Optoelectron. Adv. Mater. 10 (2008) 164
[5] F. Sanchez, H. Leblond, M. Salhi, A. Komarov, A. Haboucha, Fib. Integrat. Opt. 27 (2008) 370.

[6] V.J. Matsas, T.P. Newson, D.J. Richardson, D.N. Payne, Electron. Lett 28 (1992) 1391.

[7] N.N. Akhmediev, A. Ankiewicz, Phys. Rev. Lett. 79 (1997) 4047.

[8] A. Komarov, H. Leblond, F. Sanchez, Opt. Commun. 267 (2006) 162

[9] F. Amrani, A. Haboucha, M. Salhi, H. Leblond, A. Komarov, Ph. Grelu, F. Sanchez, Opt. Lett. 34 (2009) 2120.

[10] D.Y. Tang, W.S. Man, Y.H. Tam, P.D. Drummond, Phys. Rev. A 64 (2001) 033814

111] Ph. Grelu, F. Belhache, F. Gutty, J.M. Soto-Crespo, Opt. Lett. 27 (2002) 966.

[12] Ph. Grelu, F. Belhache, F. Gutty, J.M. Soto-Crespo, J. Opt. Soc. Am. B 20 (2003) 863

[13] A. Haboucha, H. Leblond, M. Salhi, A. Komarov, F. Sanchez, Opt. Lett. 33 (2008) 524

14] A. Komarov, A. Haboucha, H. Leblond, F. Sanchez, Opt. Lett. 33 (2008) 2254

[15] A. Haboucha, H. Leblond, M. Salhi, A. Komarov, F. Sanchez, Phys. Rev. A 78 (2008) 043806.

[16] S. Chouli, Ph. Grelu, Opt. Express 17 (2009) 11776

[17] F. Amrani, A. Haboucha, M. Salhi, A. Komarov, F. Sanchez, Appl. Phys. B 99 (2010)

[18] M. Salhi, H. Leblond, F. Sanchez, Phys. Rev. A 67 (2003) 013802. 